\title{
CUTANEOUS TUBERCULOSIS AND ITS CO-EXISTENCE WITH HIV INFECTION
}

\author{
Sunil Lad ${ }^{1}$, Lokesh Siddanan Jappa ${ }^{2}$
}

${ }_{1}^{1}$ Consultant Dermatologist, Dr. Lad's Skin Solution, Pandharpur, Maharashtra, India.

${ }^{2}$ Associate Professor, Department of Skin, STD and Leprosy, NKPSIMS Medical College and Research Institute, Nagpur, Maharashtra, India.

\section{ABSTRACT}

\section{BACKGROUND}

Cutaneous tuberculosis is one of the major chronic infective disorders of skin but now a days the disease has re-emerged in areas with a high prevalence of HIV infection.

This study was conducted to evaluate the incidence of cutaneous tuberculosis and its co-existence with HIV infection which helps in defining its status.

\section{MATERIALS AND METHODS}

20 patients clinically suspected as cutaneous tuberculosis were selected for the study over a period of one year. Detailed history, clinical examination, routine haematological test, skin biopsy, x-ray chest, Mantoux test and test for detection of HIV 1 \& 2 antibodies were carried out in all patients. Z-N staining and culture were done wherever we could obtain sample from the lesion.

\section{RESULTS}

In our longitudinal study, incidence of cutaneous tuberculosis was found to be $0.17 \%$ among patients attending OPD in tertiary care centre. The disease occurred mainly in third and fourth decade of life that is $55 \%$ of total patients. Commonly seen in Male (55\%) then female (45\%) and among these 65\% were engaged in outdoor activities, while remaining 35\% were housewives. Cutaneous tuberculosis was more common in low and middle socioeconomic class (80\%), remaining $20 \%$ were of higher class. The commonest clinical forms of cutaneous tuberculosis were lupus vulgaris (55\%) followed by scrofuloderma (25\%), tuberculosis verrucosa cutis (10\%), tuberculid (5\%) and acute miliary ТВ (5\%). In lupus vulgaris, majority of the lesions (54.55\%) over face followed by (36.36\%) over extremities and one rare case on genital region. In scrofuloderma majority of the lesions (60\%) were over cervical region. In TBVC, all the lesions were on extremities. Mantoux test was positive in (72.72\%) cases of lupus vulgaris (8 out of 11 cases), all cases of TBVC (100\%) and 20\% cases of scrofuloderma ( 1 out of 5 cases). Ziehl Neelsen staining showed acidfast bacilli in 5 cases out of 9 patients. Among these, 2 cases belonged to scrofuloderma and lupus vulgaris while 1 case was of TBVC. Culture on Lowenstein Jensen Medium was done for 6 weeks in 9 cases. Out of these, 3 cases were positive, 2 of lupus vulgaris and 1 of TBVC. X-ray chest findings suggestive of Active pulmonary Koch's in $40 \%$ of all the cases and evidence of healed lesions in $10 \%$ of cases, no abnormality was detected in remaining $50 \%$ of cases. The histopathological finding were suggestive of granuloma composed of epithelioid cells and Lymphocytes in 91\% (10 out of 11) cases of lupus vulgaris. Granuloma formation in $66.66 \%$ ( 3 out of 5) cases of scrofuloderma and 100\% in all cases of TBVC ( 2 out of 2 ). Out of remaining 2 patients, acute miliary tuberculosis and papulonecrotic tuberculid showed non-specific histopathological findings. ELISA for HIV was positive in $20 \%$ ( 4 out of 20) cases.

Limitations- 1 . Small sample size. 2 . The study is only of clinical experience of one particular area.

\section{CONCLUSION}

The diagnosis of cutaneous tuberculosis should therefore be based on history and evolution of the disease, cardinal clinical features and histopathological characteristics. An increased awareness of the re-emergence of cutaneous tuberculosis will allow for the proper diagnosis and management of this increasingly common skin disorder

\section{KEY WORDS}

Cutaneous Tuberculosis, HIV, Culture, Z-N Staining.

HOW TO CITE THIS ARTICLE: Lad S, Jappa LS. Cutaneous tuberculosis and its co-existence with HIV infection. J. Evolution Med. Dent. Sci. 2018;7(49):5281-5291, DOI: 10.14260/jemds/2018/1172

'Financial or Other Competing Interest': None.

Submission 21-10-2018, Peer Review 16-11-2018,

Acceptance 22-11-2018, Published 03-12-2018.

Corresponding Author:

Dr. Lokesh Siddanan Jappa,

Associate Professor,

Department of Skin, STD and Leprosy,

NKPSIMS Medical College and Research Institute,

Digdoh, Hingna, Nagpur,

Maharashtra, India.

E-mail: drlokeshsid@gmail.com

DOI: $10.14260 /$ jemds/2018/1172

\section{BACKGROUND}

In Dermatology Practice, Cutaneous tuberculosis has reemerged in areas with a high incidence of HIV infection and multi-drug resistant pulmonary tuberculosis.

Cutaneous tuberculosis forms one of the major chronic infective disorder of the skin. Mycobacterium tuberculosis is the predominant causative organism of cutaneous tuberculosis at times the attenuated strain of Mycobacterium bovis and the BCG vaccine may be responsible. ${ }^{1,2,3,4}$

Cutaneous infection caused by other mycobacteria like M. kansasii, M. avium, M. marinum and M. ulcerans have been documented, which at times may mimic cutaneous tuberculosis. $5,6,7$ 
It presents in variety of morphological forms. True cutaneous tuberculosis lesions can be acquired either exogenously or endogenously, these lesions include tuberculous chancre, tuberculosis verrucosa cutis, lupus vulgaris, scrofuloderma, orificial tuberculosis, miliary tuberculosis, metastatic tuberculosis abscess and most cases of papulonecrotic tuberculid. The tuberculids, like cutaneous tuberculosis, show a wide spectrum of morphology but M. tuberculosis is not identified by AFB stains, culture or PCR. These lesions include lichen scrofulosorum, nodular tuberculid, most cases of nodular granulomatous phlebitis, most cases of erythema induratum of Bazin and some cases of papulonecrotic tuberculid. ${ }^{8}$ The clinical manifestation are extremely diverse thus, a classification of cutaneous tuberculosis only on clinical ground is difficult. The diagnosis of cutaneous tuberculosis is based on relative and absolute criteria apart from other supportive immunological evidence. ${ }^{9}$

\section{Absolute Criteria}

1. Demonstration of acid-fast bacilli (AFB) on Ziehl Neelsen (ZN) staining of smear prepared with material taken from lesion.

2. Recovery of Mycobacterium tuberculosis in culture on Lowenstein Jensen's (L J ) medium after 4-6 weeks of inoculation.

3. Early detection of mycobacterial Tuberculosis in BACTEC culture by Ligase Chain reaction.

4. Animal inoculation.

5. Detection of mycobacterium antigen using radioimmunoassay or enzyme linked immunosorbent assay (ELISA) or polymerise chain reaction (PCR). ${ }^{10,11}$

6. Detection of MPT40 (specific genomic fragment of mycobacterium tuberculosis. ${ }^{12}$

7. New rapid specific and sensitive tests for detection of mycobacterial antigens are detection of MTB specific antigen by Interferon gamma release assay which are commercially available as QuantiFERON TB Gold/ Tspot.

\section{Relative Criteria}

1. Characteristic history and clinical features.

2. Mantoux test.

3. Histopathological examination of the tissue section to depict a tuberculoid or tuberculous granuloma. ${ }^{9}$

In view of the limitations of the preceding parameters the diagnosis may reasonably be made based on the history and evolution of the disease, cardinal morphological features of reinfection and reactivation tuberculosis and histopathological characteristic. A response to antitubercular drugs may be assistance. ${ }^{13}$

But now a days, the disease has re-emerged in areas with a high prevalence of Human Immunodeficiency Virus (HIV) infection. It is probably that there has been a reactivation of "persisters" in immunocompromised hosts. ${ }^{9}$

\section{Aims and Objectives}

1. To study Incidence of cutaneous tuberculosis and its coexistence with HIV infection.
2. To study the various clinical presentations and histopathological features of cutaneous tuberculosis.

\section{MATERIALS AND METHODS}

The study group included 20 patients attending outpatient department at tertiary care hospital and referral centre in south India over a period of one year. The bacteriological and histopathological study was carried out in collaboration with the Department of Microbiology and Pathology in the same institution.

All the patients of clinically suspected cutaneous tuberculosis attending skin OPD who were untreated for disease were enrolled in the study. A detailed history was elicited from every patient keeping in mind the epidemiologic features, socio-economic status as per monthly income of the family, history of intra-familial or extra familial exposure to patient of active tuberculosis. History of BCG vaccination or history of Pulmonary TB in the past along with occupation of the patient was asked. Also enquired about the initial presentation and progress of the disease, if was present. This was followed by a thorough clinical examination. The presence of active tuberculosis elsewhere in the body was sought.

Along with routine haematological tests and X-ray chest the Mantoux test was performed by injecting intradermally 1 Tuberculin unit (1TU) of commercially available tuberculin. The grading of reaction was done after 48 hours. The area of induration is measured in millimetres and positive results recorded as follows-

- $\quad<5 \mathrm{~mm}$ - Negative

- $5-10 \mathrm{~mm}-+$

- $10-20 \mathrm{~mm}-++$

- $20 \mathrm{~mm}$ or more-+++

A test for detection of HIV 1 and 2 antibodies was carried out in every patient.

Z-N staining and Culture were done wherever we could obtain sample from the lesions. We have tried to express the material from the lesion of all 20 patients but able to collect the sample from 9 patients only. Among these 9 patients 3 were Ulcerative variant of Lupus vulgaris, all 5 cases of Scrofuloderma and 1 case of TBVC.

\section{Ziehl-Neelsen Staining Procedure}

- Spread the material evenly over the central area of the slide using a continuous rotational movement.

- Then Placed slides on dryer with smeared surface upwards, and air dry for about 30 minutes. Heat Fixation of smear using burner.

- Followed by carbol fuchsin stain followed by heating until vapour starts to evaporate. Allow the heated stain to remain on the slide for 5 minutes followed by Washing off the stain with clean water.

- Followed by decolourisation of smear by acid alcohol followed by washing with clean tap water later covered by Methylene Blue stain for 5 minutes and Wash off stain with clean water followed by air drying the slide followed by examination under $100 \mathrm{X}$ oil immersion microscope. 
Preparation of LJ Medium

- $\quad$ Dissolve $37.3 \mathrm{gm}$ of the medium in $600 \mathrm{ml}$ of distilled water containing $12 \mathrm{ml}$ of glycerol.

- Heat if necessary, to dissolve the medium completely.

- $\quad$ Autoclave at $121^{\circ} \mathrm{C}$ for 15 minutes.

- Prepare $1000 \mathrm{ml}$ of a uniform suspension of fresh eggs under aseptic conditions. Avoid whipping air into suspension during the collection and mixing.

- Aseptically mix the $1000 \mathrm{ml}$ of egg suspension with 600 $\mathrm{ml}$ of the sterile Lowenstein-Jensen Medium cooled to $50^{0}-60^{\circ} \mathrm{C}$, avoiding air bubbles.

- Dispense the finished medium into sterile screw-cap test tubes.

- $\quad$ Place the tubes in a slanted position and heat at $85^{\circ} \mathrm{C}$ for 45 minutes.

Cultures should be read within 5- to 7- days after inoculation and once a week thereafter for up to 8 weeks.

Skin biopsy was done in all twenty cases. In case of lupus vulgaris, biopsy was done from the growing margin of the lesion while biopsy was done from edge of the ulcer in scrofuloderma, from marginal part in case of TBVC and from the Entire lesion for Tuberculid and Acute Miliary TB case. In addition, sections were stained by Ziehl Neelsen stain for the demonstration of tubercle bacilli.

\section{RESULTS}

In our study the Incidence of Cutaneous tuberculosis is $0.17 \%$ noticed out of total 12, 056 patients attended in OPD in tertiary care centre over a period of one year. The disease occurred mainly in third and fourth decade that is $55 \%$ of total 20 patients, which is followed by fifth and sixth decade $30 \%$ out of 20 patients. Amongst 20 patients 11 were Males (55\%) and 9 were females (45\%) as shown in (Table I)

\begin{tabular}{|c|c|c|c|c|}
\hline Age & Male & Female & Total & Percentage \\
\hline $0-9$ & - & 1 & 1 & $5 \%$ \\
\hline $10-19$ & 1 & 1 & 2 & $10 \%$ \\
\hline $20-29$ & 4 & 3 & 7 & $35 \%$ \\
\hline $30-39$ & 3 & 1 & 4 & $20 \%$ \\
\hline $40-49$ & 2 & 1 & 3 & $15 \%$ \\
\hline$>50$ & 1 & 2 & 3 & $15 \%$ \\
\hline Total & $11(55 \%)$ & 9 (45\%) & $\mathbf{2 0}$ & $\mathbf{1 0 0 \%}$ \\
\hline \multicolumn{6}{|c|}{ Table I. Relation with Age \& Sex } \\
\hline
\end{tabular}

Among these 20 patients, $13(65 \%)$ were engaged in outdoor activities (20\% were students, $25 \%$ were unskilled workers and $20 \%$ were skilled workers) while remaining $7(35 \%)$ were housewives. As shown in (Table II)

\begin{tabular}{|c|c|c|}
\hline Occupation & No. of Cases & Percentage \\
\hline Student & 4 & $20 \%$ \\
\hline Unskilled work & 5 & $25 \%$ \\
\hline Skilled work & 4 & $20 \%$ \\
\hline House wife & 7 & $35 \%$ \\
\hline \multicolumn{2}{|c|}{ Table II. Relation with Occupation } \\
\hline
\end{tabular}

Cutaneous tuberculosis was more common in low (40\%) and middle socioeconomic class $(40 \%)$ of total patients, remaining $20 \%$ were of higher class. As shown in (Table III)

\begin{tabular}{|c|c|c|}
\hline Socioeconomic Status & No. of Cases & Percentage \\
\hline $\begin{array}{c}\text { Lower }<500 \text { per month } \\
\text { per head }\end{array}$ & 8 & $40 \%$ \\
\hline Middle 500-2000 & 8 & $40 \%$ \\
\hline Higher $>2000$ & 4 & $20 \%$ \\
\hline \multicolumn{3}{|c|}{ Table III. Socioeconomic Status } \\
\hline
\end{tabular}

The commonest clinical forms of cutaneous tuberculosis were Lupus vulgaris (55\%) followed by Scrofuloderma (25\%), tuberculosis verrucosa cutis (10\%), Tuberculid (5\%) and Acute Miliary TB (5\%). As shown in (Table IV)

\begin{tabular}{|c|c|c|}
\hline Type & No. of Cases & Percentage \\
\hline Lupus Vulgaris & 11 & $55 \%$ \\
\hline Scrofuloderma & 5 & $25 \%$ \\
\hline TBVC & 2 & $10 \%$ \\
\hline Acute miliary TB & 1 & $5 \%$ \\
\hline Tuberculid & 1 & $5 \%$ \\
\hline Total & 20 & $100 \%$ \\
\hline \multicolumn{3}{|c|}{ Table IV. Incidence of the Type of Cut. TB } \\
\hline
\end{tabular}

In Lupus vulgaris, majority of the lesions (54.55\%) over face followed by (36.36\%) over extremities and one $(9.09 \%)$ rare case on penile region. As shown in (Table V)

\begin{tabular}{|c|c|c|}
\hline Site of Lesion & No. of Cases & Percentage \\
\hline Face \& Neck & 6 & $54.55 \%$ \\
\hline Trunk & - & $0.0 \%$ \\
\hline Extremities & 4 & $36.36 \%$ \\
\hline Genitalia (Penile region) & 1 & $9.09 \%$ \\
\hline Widespread lesion & - & $0.0 \%$ \\
\hline \multicolumn{2}{|c|}{ Table V. Distribution of Lupus Vulgaris according to Site } \\
\hline
\end{tabular}

In Scrofuloderma, majority of the lesions (60\%) over cervical region followed by $20 \%$ each in Axillary and inguinal lymph nodes. As shown in (Table VI)

\begin{tabular}{|c|c|c|}
\hline Site of Lesion & No. of Cases & Percentage \\
\hline Cervical lymph nodes & 3 & $60.00 \%$ \\
\hline Axillary lymph nodes & 1 & $20.00 \%$ \\
\hline Inguinal lymph nodes & 1 & $20.00 \%$ \\
\hline Table VI. Distribution of Scrofuloderma according to Site
\end{tabular}

In TBVC, all the lesions were on extremities. As shown in (Table VII)

\begin{tabular}{|c|c|c|}
\hline Site of Lesion & No. of Cases & Percentage \\
\hline Face & - & $00 \%$ \\
\hline Trunk & - & $00 \%$ \\
\hline Upper extremity & - & $00 \%$ \\
\hline Lower Extremity & 2 & $100 \%$ \\
\hline \multicolumn{2}{|c|}{ Table VII. Distribution of TBVC according to Site } \\
\hline
\end{tabular}

Mantoux test was positive in $(72.72 \%)$ cases of lupus vulgaris (8 out of 11 cases), all cases of TBVC (100\%) and (20\%) cases of scrofuloderma (1 out of 5 cases), Positive in $100 \%$ cases of PN tuberculid (1 case) and Acute Miliary tuberculosis was negative. We have considered "+" result of Mantoux test as Negative finding as shown as (Table VIII) 


\begin{tabular}{|c|c|c|c|c|c|c|c|c|c|c|}
\hline \multirow{2}{*}{ Results of the Test } & \multicolumn{2}{|c|}{ LV } & \multicolumn{2}{|c|}{ Scrofuloderma } & \multicolumn{2}{|c|}{ TBVC } & \multicolumn{2}{|c|}{ AM TB } & \multicolumn{2}{|c|}{ PN Tuberculid } \\
\hline & No. & $\%$ & No. & $\%$ & No. & $\%$ & No. & $\%$ & No. & $\%$ \\
\hline Ulcerative & - & - & 1 & 20 & - & - & - & - & - & - \\
\hline+++ & 2 & 18.2 & - & - & - & - & - & - & - & - \\
\hline++ & 6 & 54.51 & - & - & 2 & 100 & - & - & - & - \\
\hline$+/$ Negative & 3 & 27.3 & 4 & 80 & - & - & 1 & 100 & 1 & 100 \\
\hline
\end{tabular}

* LV-Lupus vulgaris, AM TB-Acute Miliary Tuberculosis, PN Tuberculin-Papulonecrotic tuberculid.

Ziehl Neelsen staining for acid fast bacilli were demonstrated in 5 cases out of 9 patients. Among these 2 cases each were belonging to scrofuloderma and lupus vulgaris while 1 case was of TBVC.

Culture on Lowenstein Jensen Medium was done for 6 weeks in 9 cases. Out of that 3 cases were positive, 2 of lupus vulgaris and 1 of TBVC.

\begin{tabular}{|c|c|c|c|c|c|c|c|}
\hline X-Ray Findings & LV & Scrof & TBVC & AM TB & PN Tuberculid & Total & \% \\
\hline Evidence of active pulmonary Kochs & 2 & 4 & - & 1 & 1 & 8 & 40 \\
\hline Evidence of healed lesion & 1 & - & 1 & - & - & 2 & 10 \\
\hline No. Abnormality & 8 & 1 & 1 & - & - & 10 & 50 \\
\hline \multicolumn{7}{|c|}{ Table IX. Chest X-Ray Findings } \\
\hline
\end{tabular}

The histopathological finding suggestive of granuloma composed of epithelioid cells and Lymphocytes in 91\% (10 out of 11) cases of lupus vulgaris. Granuloma formation in $66.66 \%$ ( 3 out of 5 ) cases of scrofuloderma and $100 \%$ in all cases of TBVC (2 out of 2). As shown in (Table X)

\begin{tabular}{|c|c|c|c|}
\hline Type & $\begin{array}{c}\text { Clinically } \\
\text { Diagnosed }\end{array}$ & $\begin{array}{c}\text { Histopathologic } \\
\text { Confirmation }\end{array}$ & $\mathbf{\%}$ \\
\hline Lupus Vulgaris & 11 & 10 & 90.90 \\
\hline Scrofuloderma & 5 & 3 & 60.00 \\
\hline TBVC & 2 & 2 & 100.00 \\
\hline Acute miliary TB & 1 & - & 0.0 \\
\hline Tuberculid & 1 & - & 0.0 \\
\hline \multicolumn{2}{|r|}{ Table X. Clinico-Histological Correlation } \\
\hline
\end{tabular}

ELISA for HIV was positive in $20 \%$ ( 4 out of 20 ) cases. As shown in (Table XI)

\begin{tabular}{|c|c|c|c|c|c|c|c|}
\hline $\begin{array}{c}\text { ELISA } \\
\text { Test for } \\
\text { HIV }\end{array}$ & LV & Scrof & TBVC & AMTB & Tuberculid & Total & $\%$ \\
\hline Positive & - & 2 & - & 1 & 1 & 4 & 20 \\
\hline Negative & 11 & 3 & 2 & - & - & 16 & 80 \\
\hline
\end{tabular}

\section{Table XI. HIV Positivity and Cutaneous TB}

The X-Ray chest findings suggestive of Active pulmonary Koch's in $40 \%$ of all the cases and evidence of healed lesions in $10 \%$ of cases, No abnormality detected in remaining $50 \%$ of cases. As shown in (Table IX)

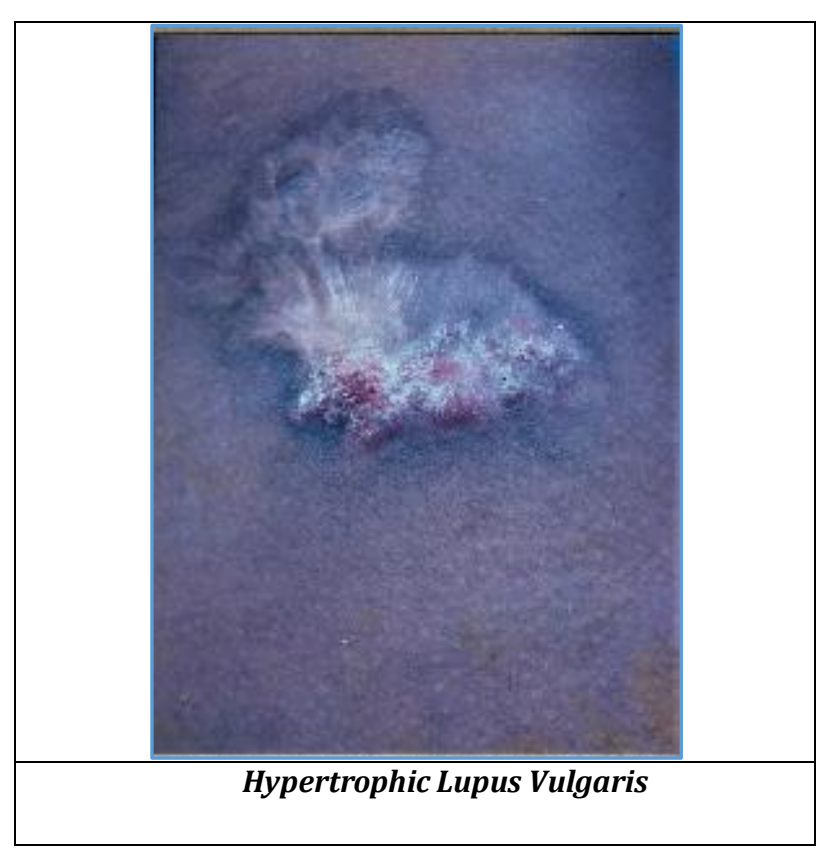



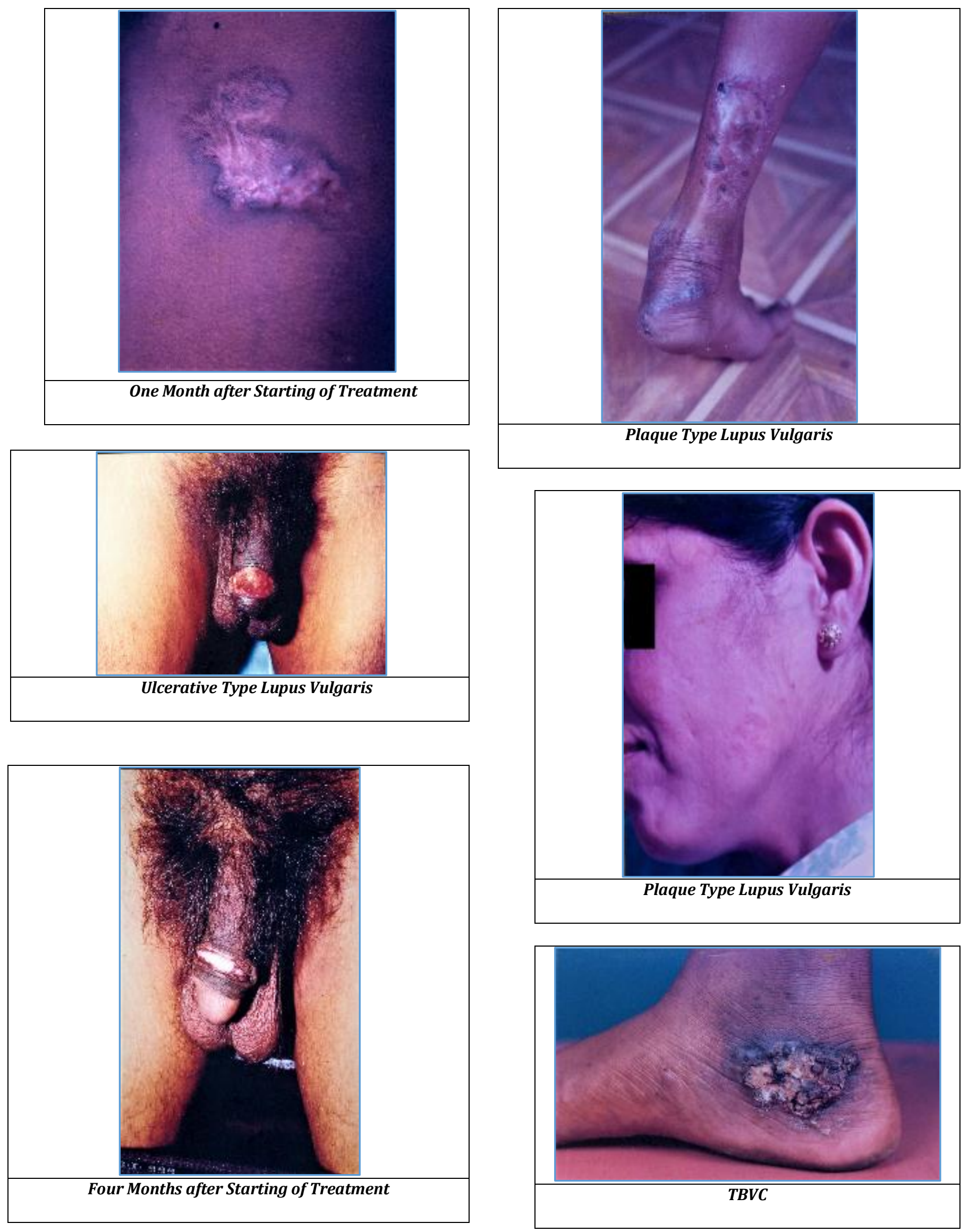

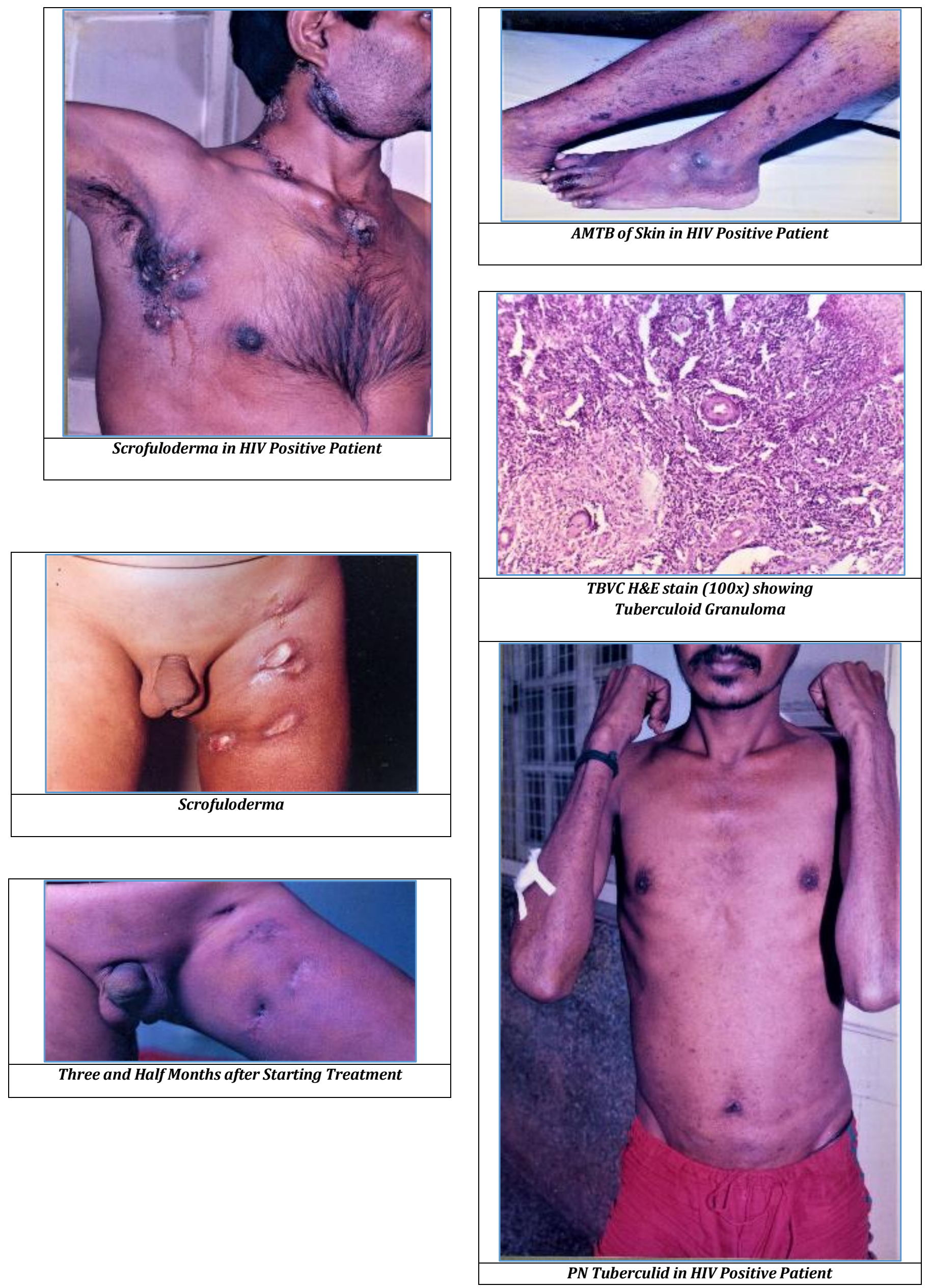

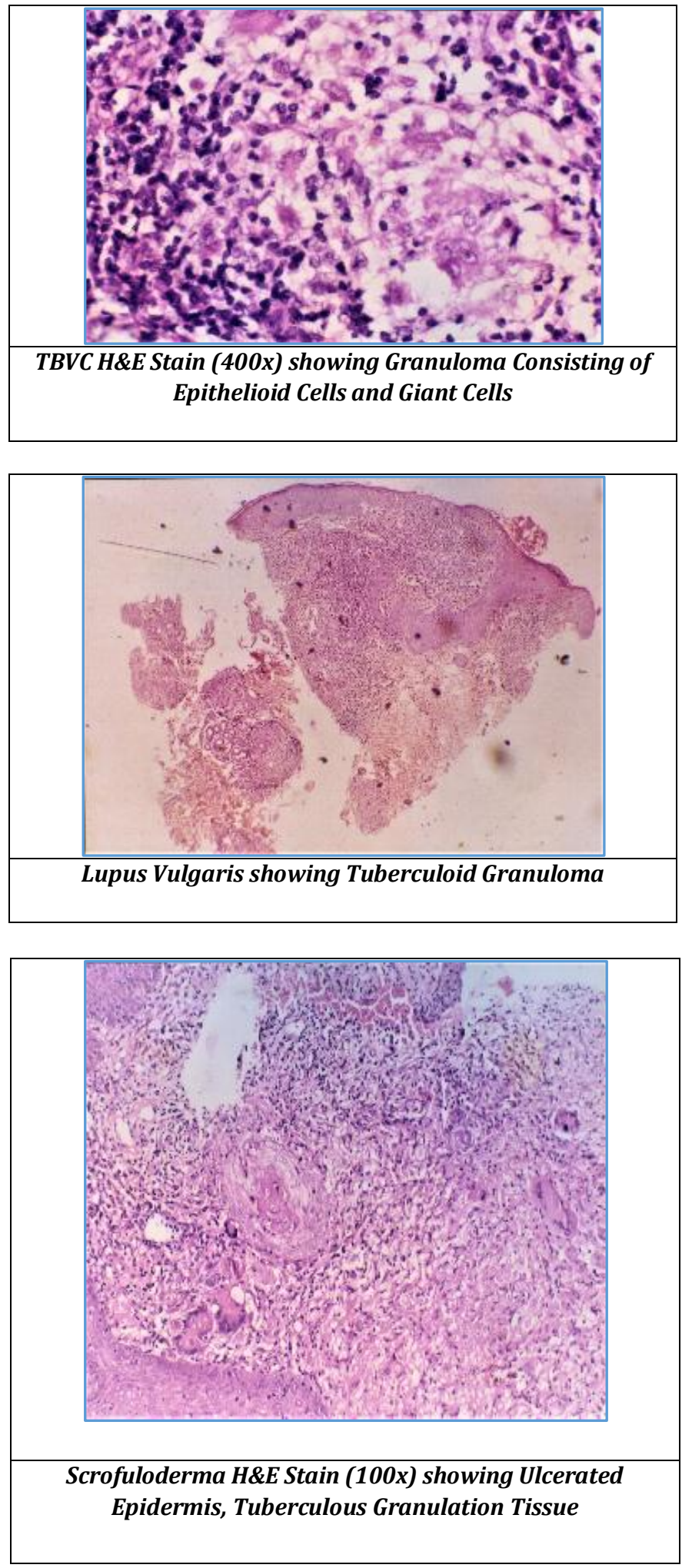

\section{DISCUSSION}

In one year period we studied 20 clinically suspected cases of cutaneous tuberculosis among the total 12056 patients who attended the Skin and STD OPD in Tertiary care centre.

Patients from all age groups were affected by skin tuberculosis $1(5 \%)$ in first decade, $2(10 \%)$ in second

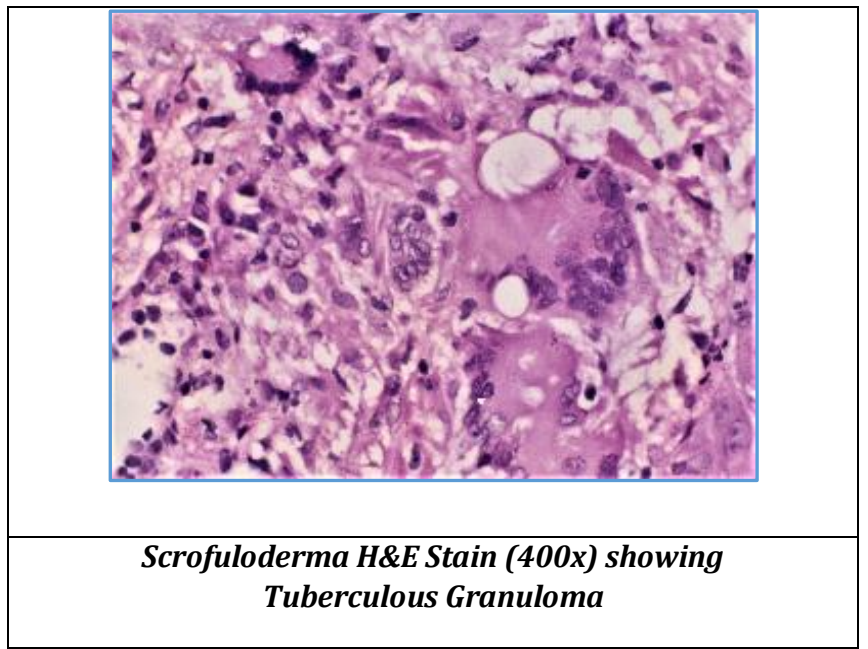

Observation Graphs

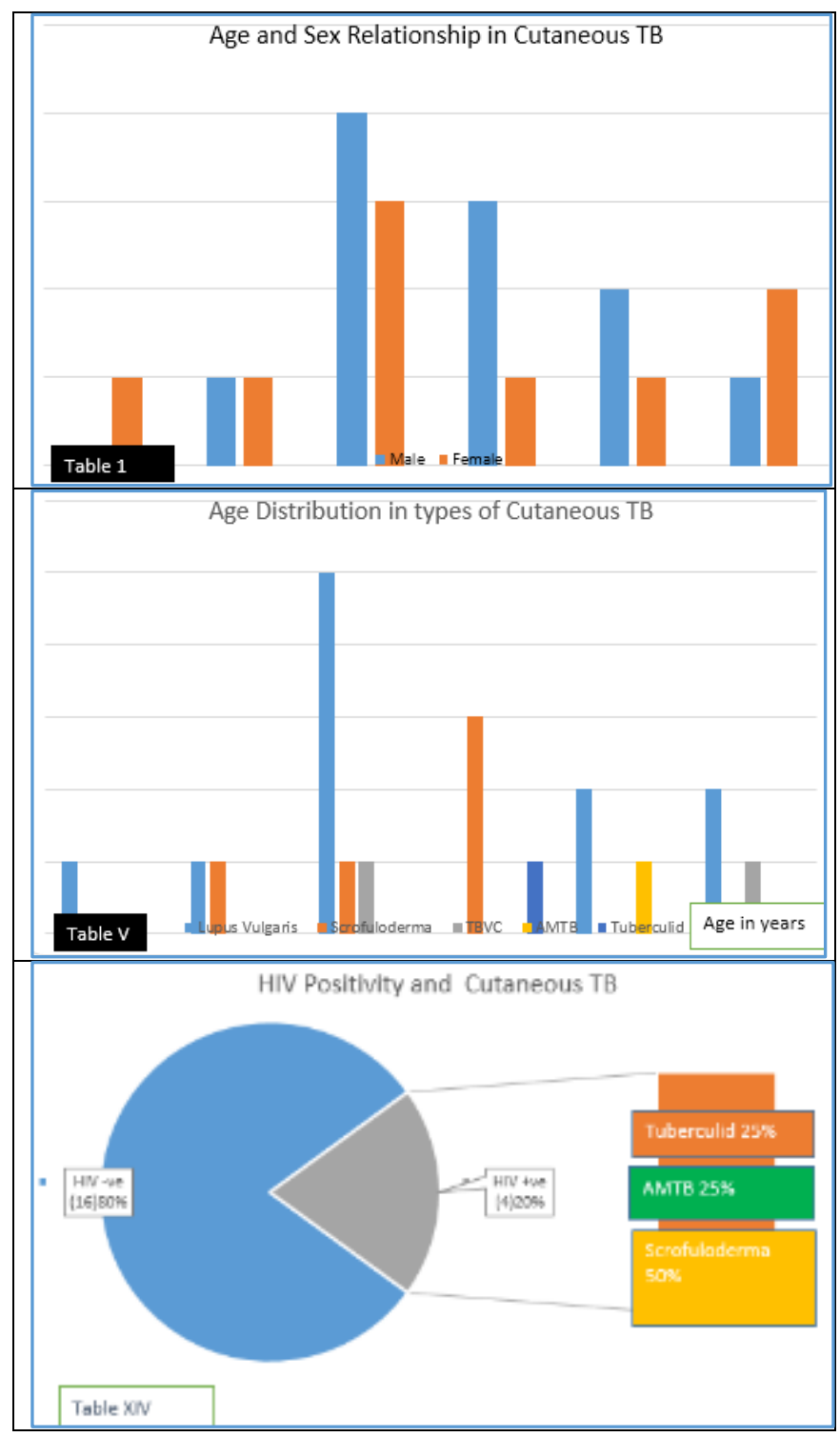

decade, 7 (35\%) in third decade, $4(20 \%)$ in forth decade and $3(15 \%)$ in fifth decade. $3(15 \%)$ patients were more than 50 years of age. Thus $13(65 \%)$ cases were between second and forth decade. Kumar B, Kaur S. observed 52 (78\%) cases between 11-40 years. ${ }^{14}$ Ramesh, Misra and Jain observed majority of patients between 11-40 years of age. ${ }^{15}$ 
Sehgal, Jain and Srivastva found $67 \%$ of the patients in age group 10-39 years. ${ }^{13}$ Our observations are almost parallel with these studies.

In studying sex distribution in cutaneous tuberculosis, we observed that 11 (55\%) were males and 9 (45\%) were females. Sehgal, Jain and Srivastva in their study observed 61.5\% male and 38.5\% female patients. ${ }^{13}$ Ramesh, Misra and Jain observed $63 \%$ males and $37 \%$ females. ${ }^{15}$ Wong K.C., Lee, et al observed equal distribution of cutaneous tuberculosis among male and female patients. ${ }^{16}$ Our findings are in accordance with those of Sehgal, Jain and Srivastva. Thus, males are predominantly affected which could be explained by their outdoor work and more exposure to environmental hazards. ${ }^{13}$

We have noticed that cutaneous tuberculosis was common in those whose occupation with more of outdoor activity, 13 (65\%) cases belongs to them.

Regarding the socioeconomic status of the patients, we have observed that cutaneous tuberculosis was common in low- and middle-income group. But 4 cases of lupus vulgaris were belonging to higher socioeconomic status.

Out of 20 cases, 11 (55\%) were lupus vulgaris, 5 (25\%) were scrofuloderma, $2(10 \%)$ were tuberculosis verrucosa cutis and one each of Acute miliary tuberculosis (5\%) and papulonecrotic tuberculid (5\%). Kumar B, Kaur Surinder in the period of four years found 66 cases. ${ }^{7}$ Cut TB out of 39074 dermatological patients, giving an incidence of $0.17 \%$. They found $54(81 \%)$ cases of lupus vulgaris, $14(21 \%)$ cases of scrofuloderma, 6 (9\%) cases of TBVC, our findings are similar to Kumar B, Kaur S. ${ }^{14}$

Ramesh, Misra and Jain in their study observed lupus vulgaris (59\%), scrofuloderma (27\%) and tuberculosis verrucosa cutis (14\%). ${ }^{15}$ The findings in our study are similar to those of Ramesh, Misra and Jain. There is higher incidence of pulmonary tuberculosis in our community and BCG is an important component of immunisation programme for control of tuberculosis. That is why there is better herd immunity and hypersensitivity against tuberculosis. So lupus vulgaris is the commonest form of cutaneous tuberculosis in our community.

In studying the Distribution of lupus vulgaris according to the site, we noticed that out of 11 cases of lupus vulgaris, 6 (54.55\%) had lesions over face and neck. 4 (36.36\%) had lesions over lower extremities and 1 (9.1\%) had lesion over penis which is a very rare presentation earlier reported by $\mathrm{C}$. Gangalakshmi and Sankaramahalingam. ${ }^{17}$

Marcoval J., Servitje 0 et al in their study of 10 cases of lupus vulgaris noticed around 6 cases with lesion over face. 18 Kumar B, Kaur S in their study of 6 cases found, face and neck as the commonest site in 23 (35\%) patients followed by extremities in $20 \%$ cases.

In our study lupus vulgaris was found most commonly over face and neck followed by extremities.

In studying morphological variant of lupus vulgaris, we observed that out of 11 cases 7 (63.63) cases were plaque type, $3(27.3 \%)$ cases were ulcerative type and 1 (9.1\%) case was hypertrophic type of lupus vulgaris. ${ }^{19}$

In the present study out of 5 patients of scrofuloderma 3 $(60 \%)$ patients had involvement of cervical lymph nodes, 1 (20\%) had axillary and 1 (20\%) had inguinal lymph node involvement. Sehgal, Jain and Srivastva in their study of 47 cases of scrofuloderma, noted 15 cases with cervical lymph node involvement, 7 with axillary, 6 with inguinal, 4 with parasternal, 2 with retroauricular lymph node involvement. ${ }^{13}$

Satyanarayan in his study observed neck and axillae as commonest location of scrofuloderma. The Upper cervical group was involved more frequently than then other groups. ${ }^{20}$ Our findings are similar to that of Sehgal, Jain, Srivastava and of Satyanarayana.

One patient of scrofuloderma in our study had concomitant lepromatous leprosy and morphoea (proved by histopathologically) which is a very rare combination.

Out of 2 cases of TVC 2 (100\%) had lesion over lower extremity. In lower extremity site of predilection was dorsum of foot. Singh $\mathrm{n}$ his study of 10 cases noticed 7 with lesions over lower extremity and 3 with lesions over hands. ${ }^{21}$

Ramesh, Misra and Jain in their study observed that out of 14 patients of TBVC 10 had lesions over lower extremity and 3 had lesions over upper extremity and single patient had a lesion over buttock. ${ }^{15}$ Our findings are consistent with Singh, Ramesh, Misra and Jain. Most of the Indian people work barehanded and walk barefoot, so extremities are more prone to injuries. This may be the reason that all the lesions of TBVC were observed over extremities.

We have studied one case of Acute miliary tuberculosis of the skin who was HIV positive, patient had crusted and ulcerative lesions over the extremities with pulmonary tuberculosis. Kennedy C, and Sehgal, Wagh have also noted similar clinical features in cases of Acute miliary tuberculosis 22,23

One case of papulonecrotic tuberculid was observed during the study period. Patient also had pulmonary tuberculosis. Bilaterally symmetrical hyperpigmented papules, crusted and necrotic lesions were present over extremities and trunk. Our findings are consistent with Morrison J. G., Fourie E. D. 24

In studying relation to cutaneous tuberculosis with systemic tuberculosis, we observed the active focus of tuberculosis on X-Ray chest in 4 cases of scrofuloderma, 2 cases of lupus vulgaris and one case each of Acute miliary tuberculosis of skin and papulonecrotic tuberculid. Evidence of healed lesion of tuberculosis was seen in one case each of lupus vulgaris and TBVC.

The results of the Mantoux test are depicted in Table XI. Out of 11 cases of lupus vulgaris, $2(18 \%)$ cases showed $(+++)$ response, $6(54.5 \%)$ cases showed $(++)$ response, $2(18 \%)$ cases showed $(+)$ response and $1(9 \%)$ case showed negative response to the test. Out of 5 cases of scrofuloderma $1(20 \%)$ case showed ulcerative reaction to the test, $2(20 \%)$ cases showed (+) response and $2(40 \%)$ cases which were HIV positive showed negative response to the test. Out of 2 cases of TBVC $2(100 \%)$ cases showed (++) response to the test. One case of papulonecrotic tuberculid showed $(+)$ response to the test and one case of Acute miliary tuberculosis of skin showed negative response to the test. These two patients were HIV positive.

In our study we found negative Mantoux in $4(20 \%)$ cases. Kumar B, Kaur S in their study found negative Mantoux test in $(27 \%)$ cases. $^{14}$ Sehgal, Jain and Srivastva in their study noticed moderate $(++)$ to high $(+++)$ response in both 
reactivation and reinfection type of cutaneous tuberculosis, while 5 scrofuloderma out of 33 showed mild (+) response and 2 out of 7 cases of TBVC showed mild (+) response. Satyanarayana observed positive response to tuberculin test in all the cases. ${ }^{20}$

Our findings are close to Sehgal, Jain and Srivastva. As there is higher cell mediated immunity and hypersensitivity response in lupus vulgaris strongly positive Mantoux test is seen in lupus vulgaris in comparison with other form of cutaneous tuberculosis.

Out of 20 cases in our study 16 were BCG vaccinated while in rest of 4 cases BCG scar was absent.

On Ziehl Neelsen staining acid-fast bacilli were demonstrated in 5 (25\%) cases. Out of 5, 2 cases each were belonging to scrofuloderma and lupus vulgaris while 1 case was of TBVC.

Culture on Lowenstein Jensen Medium was done for 6 weeks in 9 cases. out of that 3 cases were positive, 2 of lupus vulgaris and 1 of TBVC.

In studying histopathologic features of cutaneous tuberculosis, it was observed that, out of 11 cases of lupus vulgaris histopathologic confirmation were obtained in 10 (90.90\%) cases. Out of 2 cases of TBVC 2 cases showed histopathologic picture consistent with clinical diagnosis. 3 out of 5 patients of scrofuloderma showed histopathologic picture consistent with the clinical findings.

One case each of lupus vulgaris, acute miliary tuberculosis and papulonecrotic tuberculid showed non-specific histopathology showing lymphocytes and plasma cell infiltrate.

In 2 cases of scrofuloderma acid fast bacilli were demonstrated. Kumar B, Kaur S observed non-specific picture in $18.2 \%$ cases, out of total 66 cases. Ramesh, Misra and Jain in their study of 100 cases observed non-specific picture in 18 cases. They observed dermal infiltrate of chronic inflammatory cells. Our findings are similar to Kumar B, Kaur S, Ramesh, Misra and Jain.

Out of 10 cases of lupus vulgaris which were confirmed histopathologically, acanthosis was seen in $4(40 \%)$ cases, papillomatosis in $2(20 \%)$, atrophic epidermis in $5(50 \%)$ and ulceration in $3(30 \%)$ cases. Sehgal et al in their study of histopathology of lupus vulgaris observed acanthosis in $41.12 \%$ cases, thinning of or atrophic epidermis in $47.10 \%$ and normal epidermis in $11.8 \%$ cases. ${ }^{19}$ Sehgal, Jain and Srivastva in their study of histopathology of 30 cases of lupus vulgaris noticed acanthosis in $40 \%$ of cases, atrophic epidermis in $36.6 \%$ cases and normal epidermis in $10 \%$ cases. Our findings are close to both the studies.

Tuberculous granuloma was observed in $6(60 \%)$ cases and tuberculoid granuloma in $4(40 \%)$ cases of lupus vulgaris. Granuloma was located in upper dermis in $6(60 \%)$ cases, extending over upper and mid dermis in $2(20 \%)$ cases and extending throughout dermis in 2 (20\%) cases. Granuloma were consisting of epithelioid cells and lymphocytes in all $10(100 \%)$ cases with Langhans giant cell in $7(70 \%)$ cases. Sehgal, Jain and Srivastava noticed tuberculous granuloma in $66.66 \%$ cases and tuberculoid granuloma in $33.33 \%$ cases. $60 \%$ of granuloma were located in upper dermis. Granulomas were mainly composed of epithelioid cells and lymphocytes. Giant cells were seen in some granulomas. ${ }^{13}$ Marcoval J., Servitje 0 et al in their study noticed tuberculous granuloma in 30\% cases, tuberculoid granuloma in $40 \%$ cases and diffuse infiltrate in $30 \%$ cases. $^{18}$ Our findings are similar to Sehgal, Jain and Srivastava. Location of granuloma in upper dermis and few giant cells in granuloma suggest an augmented cell medicated immunity in lupus vulgaris.

In studying histopathology of 3 cases of scrofuloderma, we noticed epidermal changes as acanthosis in $3(100 \%)$ cases, ulceration in $2(66.66 \%)$ cases and atrophy in 1 (33.33\%) case. Tuberculous granuloma in 2 (66.66\%) and tuberculoid granuloma in $1(33.33 \%)$ case was seen. Granulomas were confined to lower dermis in 1 (33.33\%) case and extending over mid and lower dermis in 2 (66.66\%) cases. Sehgal, Jain and Srivastava in their study noticed tuberculous granuloma in $54.55 \%$ cases and tuberculoid granuloma in $45.45 \%$ cases. Most of the granulomas were located in mid and lower dermis. Granulomas were composed of epithelioid cells and Langhans giant cells in all 3 (100\%) cases, while lymphocytes were present in $2(66.66 \%)$ cases.

Relative low percentage of lymphocytes and more Langhans cells represents lower immunity in scrofuloderma.

Biopsy study of 2 cases of TBVC was consistent with clinical diagnosis. Epidermal changes were hyperkeratosis, acanthosis and papillomatosis in both the cases.

Both the specimens showed tuberculous granuloma extending from upper to mid dermis. Sehgal, Jain and Srivastava noticed tuberculous granuloma in all 7 cases of TBVC. Our findings are consistent with them.

After pre-test counselling and consent, ELISA for HIV infection was done in all cases of cutaneous tuberculosis. 2 cases of scrofuloderma and 1 case each of Acute miliary tuberculosis and papulonecrotic tuberculid were found to be HIV positive.

\section{CONCLUSION}

1. Incidence of cutaneous tuberculosis: In our study we found an incidence rate of $0.17 \%$ of cutaneous tuberculosis among all patients attending Skin and STD Department.

2. Age: Maximum number of patients (65\%) were in second to fourth decade.

3. Sex: $11(55 \%)$ were male and 9 (45\%) were female.

4. Occupation: $65 \%$ of the total patients were engaged in outdoor activities.

5. Incidence of different types of cutaneous tuberculosis: Lupus vulgaris (55\%) is the most common type, followed by scrofuloderma $(25 \%)$ followed by tuberculosis verrucosa cutis (10\%), followed by tuberculid (5\%) and acute miliary TB (5\%)

6. Summary of clinical features:

a. Lupus vulgaris: We observed that majority of lesions $(54.55 \%)$ were over the face, followed by (36.36\%) extremities and one rare presentation of genital lupus vulgaris. Plaque type variety of lupus vulgaris was commonest and apple-jelly nodules could not be demonstrated. 
b. Scrofuloderma: It was noticed more commonly over cervical region (60\%).

c. TBVC: All the lesions of TBVC were over extremities.

d. We studied a rare combination of scrofuloderma, lepromatous leprosy with type II reaction and localised morphoea in a single case.

7. Mantoux test: It was positive (more than ++) in (72.72\%) cases of lupus vulgaris, $(100 \%)$ cases of TBVC and (20\%) cases of scrofuloderma. Ulcerative response was present in one case of scrofuloderma.

8. Clinico-histopathological correlation.

a. In $91 \%$ cases, a granuloma composed of epithelioid cells, Langhans giant cells and lymphocytes were seen. Whereas in $55.55 \%$ cases caseation necrosis was observed. In $16 \%$ cases histopathologic picture was non-specific with a dermal infiltrate of chronic inflammatory cells.

b. In most (60\%) cases of lupus vulgaris, granuloma was occupying upper dermis only. Granulomas were in lower and mid dermis in $(66.66 \%)$ cases of scrofuloderma and were in mid and upper dermis in both $(100 \%)$ cases of TBVC.

c. Acid fast bacilli could not be demonstrated in any histopathologic section on Ziehl-Neelsen staining.

9. Ziehl-Neelsen staining was done with the smear prepared from material taken from the lesion in 9 cases. Acid fast bacilli were demonstrated in 5 cases.

10. Culture of the material from the lesion was done on Lowenstein-Jensen medium for 6 weeks in 9 cases. Mycobacteria were grown in 3 cases.

11. ELISA for HIV was positive in $4(20 \%)$ cases. Two cases of scrofuloderma and one case each of acute miliary tuberculosis and papulonecrotic tuberculid found to be HIV positive.

The diagnosis of cutaneous tuberculosis should therefore be based on history and evolution of the disease, cardinal clinical features and histopathologic characteristics.

But now a days, the disease has re-emerged in areas with a high prevalence of Human Immunodeficiency Virus (HIV) infection. It is probably that there has been a reactivation of "persisters" in immunocompromised hosts. Alternatively, or in addition, impaired cellular immunity and immunologic energies have facilitated infection with low virulence mycobacteria. ${ }^{9}$

\section{REFERENCES}

[1] High WA, Evans CC, Hoang MP. Cutaneous miliary tuberculosis in two patients with HIV infection. J Am Acad Dermatol 2004;50(Suppl 5):S110-S13.

[2] Figueiredo A, Poiares-Baptista A, Branco M, et al. Papular tuberculids post-BCG vaccination. Int J Dermatol 1987;26(5):291-4.
[3] Kanwar AJ, Kaur S, Bansal R, et al. Lupus vulgaris following BCG vaccination. Int J Dermatol 1988;27(7):525-6.

[4] Marcussen PV. Lupus vulgaris following BCG vaccination. Br J Dermatol 1954;66(4):121-8.

[5] Callahan EF, Licata AL, Madison JF, et al. Cutaneous Mycobacterium kansasii infection associated with a papulonecrotic tuberculid reaction. J Am Acad Dermatol 1997;36(3 Pt 1):497-9.

[6] Cross GM, Guill MA, Aton JK. Cutaneous mycobacterium szulgai infection. Arch Dermatol 1985;121(2):247-9.

[7] Williams JT, Pulitzer DR, DeVillez RL. Papulonecrotic tuberculid secondary to disseminated mycobacterium avium complex. Int J Dermatol 1994;33(2):109-12.

[8] Savin JA. Mycobacterial infections. Chapter - 24. In: Champion RH, Burton JL, Ebling FJ, eds. Text book of Dermatology. Oxford: Blackwell Sci Publication 1992: p. 1036-56.

[9] Sehgal VN. Cutaneous tuberculosis. Dermatol Clin 1994;12(4):645-53.

[10] Patel RJ, Fries JW, Piessens WF, et al. Sequence analysis and amplification by polymerase chain reaction of a cloned DNA fragment for identification of mycobacterium tuberculosis. J Clin Microbiol 1990;28(3):513-8.

[11] Yanez MA, Coppola MP, Russo DA, et al. Determination of mycobacterial antigens in sputum by enzyme immunoassay. J Clin Microbiol 1986;23(5):822-5.

[12] Portillo DP, Murillo LA, Patarroyo ME. Amplification of a species-specific DNA fragment of mycobacterium tuberculosis and its use in diagnosis. J Clin Microbiol 1991;29(10):2163-8.

[13] Sehgal VN, Jain MK, Shrivastava G. Changing pattern of cutaneous tuberculosis. Int J Dermatol 1989;28(4):231-6.

[14] Kumar B, Kaur S. Pattern of cutaneous tuberculosis in North India. Ind J Dermatol Venereol Leprol 1986;52(4):203-7.

[15] Ramesh V, Misra RS, Jain RK. Secondary tuberculosis of the skin. Clinical features and problems in laboratory diagnosis. Int J Dermatol 1987;26(9):57881.

[16] Wong KO, Lee KP, Chiu SF. Tuberculosis of the skin in Hongkong. A review of 160 cases. Br J Dermatol 1968;80(7):424-9.

[17] Gangalakshmi C, Sankaramahalingam. Tuberculosis of glans penis - a rare presentation. J Clin Diag Res 2016;10(12):PD05-PD06.

[18] Marcoval J, Servitje O, Moreno A, et al. Lupus vulgaris. Clinical, histopathologic and bacteriologic study of 10 cases. J Am Acad Dermatol 1992;26(3 Pt 2):404-7.

[19] Sehgal VN, Srivastava G, Khurana VK, et al. An appraisal of epidemiologic, clinical, bacteriologic, histopathologic and immunologic parameters in cutaneous tuberculosis. Int J Dermatol 1987;26(8):521-6. 
[20] Satyanarayana BV. Tuberculoderma, a brief review together with statistical analysis and observations. Ind J Dermatol Venereol Leprol 1963;29(1):25-42.

[21] Singh G. Lupus vulgaris in India. Ind J Dermatol Venereol Leprol 1974;40(6):257-60.

[22] Kenney C, Knowles GK. Miliary tuberculosis presenting with skin lesions. Br Med J 1975;3(5979):356.
[23] ehgal VN, Wagh SA. Cutaneous tuberculosis. Current concepts. Int J Dermatol 1990;29(4):237-52.

[24] Morrison JG, Fourie ED. The papulonecrotic tuberculide. From Arthus reaction to lupus vulgaris. $\mathrm{Br}$ J Dermatol 1974;91(3):263-70. 